\title{
Index of Subjects
}

Adaptive optics

Astrometry

Atmospheric fluctuations

Beam combiner

Bispectrum

Calibration

Circumstellar material

Closure phase

Control system

Diffraction

Dispersion

Dust

Fried parameter $r_{0}$

Fringe detection and tracking

Guiding system

Image processing

Interference

astrometric

broad band

field of view

from space

heterodyne

intensity

optical, multi-aperture

pupil-plane

using radar

single aperture

speckle

Ionosphere

Isoplanatic patch

Laser guide stars

Limiting sensitivity and $\mathrm{S} / \mathrm{N}$

Magnetoplasma

Metrology

Microlensing

Modulation transfer function

Non-redundant masking

Occultation methods
$168,273,283,293,297,302,305,308,488$

$121,313,345,423,427,437,445,473$

$2,19,29,101,106,221,239,258,299,349,420,470,483$

$84,139,148,152,155,164,177,264,314,431,486$

$91,187,209,297,319,346,373,472$

$41,102,105,119,128,239,265,322,344,349,352,368,462$

$27,74,336,387,391$

$164,178,188,297,340,344,445$

$172,181,185,258$

224

$138,156,228,263,313$

$27,355,366,383,416$

$2,87,139,164,199,277,306,308,373,398,470$

$12,139,156,159,166,172,184,257,312,392,419,434$

$164,172,275$

$39,62,91,129,157,197,205,210,212,215,222$, $247,318,346,367,392,422$

$22,174,427,448,474,480$

$80,121,313,327,331,439$

86,221

$193,473,479,483$

$19,257,383$

339

$83,135,143,151,155,163,167,171,174,177,187$, $265,331,407,410,413,427,469,485$

197,419

457

$297,317,327,371,395$

$205,209,317,346,364,373,379,391,398,416$

$243,339,439,446$

221

$278,283,293,470$

$75,93,95,120,128,153,244,275$,

$285,319,343,368,472,478$

361

$159,174,178,259,431,488$

451

62,197

$296,317,327,395$

$71,364,376,416$ 
Optical fibres

Partially redundant masking

Path length compensation

Polarization

Quantum phenomena

Radio arrays

Radio correlator

Random-walk turbulence

Redundant spacing calibration

Seeing

Site testing

Speckle masking

Strehl ratio

Structure function

Super-resolution

Variable radio sources

Visibility loss

Wavefront sensor
$227,238,261,349,392$

296,323

$138,147,152,166,172,184,187,194,238,266,431$

$95,262,361,457$

11,311

$31,89,95,101,105,109,117,125,128,131,227,235,243$, $311,331,334,337,343,349,352,367,445,477,493$

33

299

187,319

$1,21,140,241,299,302,421$

$29,102,240$

$296,347,398$

$275,294,306$

$2,101,438$

218

201

225

$276,289,293,304,308$ 